Chirurg 2013 $\cdot 84: 523$

DOI 10.1007/s00104-013-2523-y

Online publiziert: 28. April 2013

(c) Springer-Verlag Berlin Heidelberg 2013
H. Dralle - I. Satiroglu

Klinik für Allgemein-, Viszeral- und Gefäßchirurgie, Medizinische Fakultät, Universitätsklinikum Halle

\section{Ileojejunale neuroendokrine Tumoren}

\section{Daran denken!}

\section{Originalpublikation}

Habbe N, Fendrich V, Heverhagen A et al (2012) Outcome of surgery for ileojejunal neuroendocrine tumors. Surg Today (Epub ahead of print). DOI 10.1007/s00595-012-0408-1

\section{Hintergrund}

Seit Oberndorfer (1907, [1]) gehören neuroendokrine Tumoren des Jejunum und Ileum wegen der Häufigkeit ihres Vorkommens im Gastrointestinaltrakt (25-30\%) zu den am besten untersuchten neuroendokrinen Neoplasien (NEN). Da es sich vielfach um kleine, frühzeitig metastasierte Tumoren handelt, die auch modernen Bildgebungsverfahren nicht selten entgehen, ist das „Daran-Denken“ besonders wichtig - gerade auch für Chirurgen, wenn es darum geht, einen bislang unbekannten Primärtumor bei abdominellen, meist hepatischen Metastasen zu sichern.

\section{Methoden}

Die vorliegende Untersuchung analysiert retrospektiv die anamnestischen, bildgebenden, operativen und Nachuntersuchungsbefunde von 97 zwischen 1999 und 2010 behandelten Patienten mit ileojejunalen NEN. Das mediane Follow-up der Patienten betrug 42 (1-132) Monate.

\section{Ergebnisse}

Insgesamt 78\% der Patienten waren symptomatisch (unspezifische Bauchschmer- zen, Darmobstruktion, Karzinoidsyndrom), 6\% hatten seit mehr als 10 Jahren uncharakteristische Bauchbeschwerden. Bei 15\% wurden die ileojejunalen NEN als Zufallsbefund einer Koloskopie diagnostiziert. $63 \%$ hatten initiale, meist hepatische Fernmetastasen. Nur bei 55\% der Patienten konnte der Primärtumor bildgebend dargestellt werden. Fast alle Tumoren lagen im Ileum (97\%), kein Patient hatte sowohl NEN im Ileum als auch im Jejunum. $24 \%$ hatten multifokale NEN, in keinem dieser Fälle wurde die Multifokalität bildgebend entdeckt. Der für das aktuelle Grading wesentliche Ki67-Index hatte für das vorliegende Kollektiv keine verwertbare Bedeutung, da 93\% der Tumoren G1-Tumoren (Ki67-Index $\leq 2$ ) waren. Multivariat waren Fernmetastasen (HR 5,39) und inkomplette Resektion (HR 2,87) die ausschlaggebenden Prognosefaktoren.

\section{Diskussion und Fazit}

Die sorgfältig durchgeführte Studie resümiert folgende aktuell wichtige Schlussfolgerungen:

- da auch moderne Bildgebungsverfahren oft nicht zielführend sind, ist das "Daran-Denken" entscheidend; die Koloskopie kann bei Intubation des terminalen Ileums die Diagnose sichern;

- ileojejunale NEN eignen sich nicht zur laparoskopischen Diagnostik und Resektion, da sie häufig sehr klein sind, in einem Viertel der Fälle multi- pel und in ca. $30 \%$ bereits lymphogen metastasiert;

- der Ki67-Index ist aufgrund der vorliegenden Untersuchung gerade bei den am häufigsten metastasierten gastrointestinalen NEN offenbar von untergeordneter Bedeutung; dieser Befund sollte zu weiteren kritischen Untersuchungen dieses für das aktuelle Grading wesentlichen Prognoseparameters führen.

\section{Korrespondenzadresse}

Prof. Dr. Dr. h.c. H. Dralle

Klinik für Allgemein-,

Viszeral- und Gefäßchirurgie,

Medizinische Fakultät,

Universitätsklinikum Halle,

Ernst-Grube-Str. 40, 06097 Halle

henning.dralle@uk-halle.de

Interessenkonflikt. Der korrespondierende Autor gibt für sich und seinen Koautor an, dass kein Interessenkonflikt besteht.

\section{Literatur}

1. Oberndorfer $S$ (1907) Karzinoide Tumoren des Dünndarms. Frankf Z Pathol 1:425-432 